\title{
Assessment of Aliphatic Based Soot Inception in Laminar Diffusion Flames
}

\author{
Nemanja Ceranic, Seth B. Dworkin \\ Department of Mechanical and Industrial Engineering \\ Ryerson University \\ Toronto, Canada
}

\begin{abstract}
Soot models are key components of computation fluid dynamic combustion codes that attempt to prescribe how soot is formed. However, due to the complex nature of soot formation, not all pathways may have been fully characterized. This work investigates numerically the influence that an aliphatic-collision (open-chain hydrocarbon) based soot inception model has on soot formation for coflow ethylene/air and methane/air laminar diffusion flames. In the literature, prediction of the soot volume fraction along the centerline of coflow ethylene flames is lacking in accuracy. Similarly for methane flames, soot formation on the wings are under predicted by many models. A new collision based inception model has been developed for specific aliphatics, and applied using an existing framework for molecular collision, in conjunction with pyrene based inception. The purpose of this model is not to be completely fundamental in nature, but more so a proof of concept in that by using physically realistic values for surface reactivity and collision efficiency, this collision mechanism can account for soot formation deficiencies that exist with just polycyclic aromatic hydrocarbon (PAH) based inception. Using this new model, the peak soot volume fraction along the centerline of an ethylene flame can be increased while the peak soot volume fraction along the wings remains unchanged, showing potential to significantly improve the model's predicative capability. Applying this model to a methane flame has resulted in an increase in the soot volume fraction in both the centerline and the wings, again improving predictive capability.
\end{abstract}

\section{INTRODUCTION}

Any form of hydrocarbon combustion leads to the generation of soot. These particles are hazardous to human health $[1,2]$ as well as dangerous for the environment [3-5]. Thus it is necessary that the characterization of soot formation be well understood in order to design more environmentally friendly combustion devices. Numerical combustion models require extensive knowledge of soot formation in order to obtain meaningful results. One of the advantages that numerical modelling can provide is that it allows researchers to test new theories at virtually no cost compared to experimental techniques.

Soot formation is a complex process consisting of polycyclic aromatic hydrocarbon (PAH) gas-phase growth, particle nucleation, surface growth via surface reaction and $\mathrm{PAH}$ condensation, surface oxidation, particle coalescence, particle coagulation and fragmentation, gas-phase scrubbing, and radiation [6]. These pathways are widely considered by the scientific community to be the accepted routes of soot formation, however, due to its complex nature, other pathways may exist that have yet to be fully characterized.

Soot models have been the subject of discussion for several decades and continue to improve to this day. The three classifications of models are empirical, semi-empirical, and predominantly fundamental models. Semi-empirical soot models [7, 8] may have a foundation in the physics behind soot formation, but lack the fundamental physics behind the problem as they rely on tunable parameters. Although these models may not accurately portray what is occurring inside of the flame, they do give insight as to which parameters may be correlated. They are an essential milestone towards the creation of a fundamental model.

One such model is the Hydrogen-Abstraction-CarbonAddition (HACA) growth mechanism introduced by Frenklach and Wang [9]. This model traditionally uses surface reactivity $(\alpha)$ as a tunable constant or function for matching numerical peak soot predictions to experimental peaks [9]. Therein lies the issue that current soot models face, which is, although peak soot may be correctly predicted, other regions of the flame may not be correctly predicted through the same tuning of $\alpha$. It has been postulated that the centerline of coflow laminar diffusion flames are dominated by inception whereas the wings are dominated by surface growth through the HACA mechanism [10]. By tuning $\alpha$ to experimental peaks, which occur along the wings, the prediction in the wings region may be corrected. However, many models do not have sufficient reaction chemistry or appropriate sub mechanisms to accurately capture the trends along the centerline, and tend to under predict soot formation in that region [11-14].

Based on recent literature, soot is able to undergo mass growth in the absence of gas phase hydrogen atoms and nascent soot can be rich in aliphatic molecules in premixed flames [15]. This observation indicates that the HACA mechanism is not likely to be responsible for this growth. The HACA mechanism removes hydrogen from the surface of molecules meaning that gas phase hydrogen atoms would be present 
whenever this mechanism is active. If the atoms are not present, another mechanism is responsible for this growth. It is proposed that aliphatic-collision based inception may have some influence on this soot mass growth. A semi-empirical model has been generated to investigate the impact that additional aliphatic collision based inception in the soot formation model would have on a variety of coflow laminar diffusion flames. More details on the physics and assumptions of the model will be explained in the following sections. The model is applied to both pure ethylene/air and methane/air laminar diffusion flames that use the same burner dimensions. Two parameters are key to this model which are the surface reactivity, $\alpha$, and the collision efficiency, $\beta$.

\section{BURNER AND NUMERICAL MODEL}

CoFlame is the in-house FORTRAN code that this research implements, which was recently formalized and published in Eaves et al. [16]. The code has been parallelized to reduce computational cost and has been validated against ethylene/air and methane/air combustion [16]. The first flame that is simulated is a coflow ethylene/air laminar diffusion flame that was originally studied by Santoro et al. [17, 18]. In those works, Santoro and coworkers had conducted experiments for several flames, four of which were pure ethylene/air diffusion flames. Of those flames, the one of particular interest is the second non-smoking flame (NSII) due to its prevalence in the literature and abundance of experimental data [19-22]. The NSII has a fuel velocity of $3.98 \mathrm{~cm} / \mathrm{s}$ and an air velocity of 8.9 $\mathrm{cm} / \mathrm{s}$ [18]. The burner consists of an $11.1 \mathrm{~mm}$ diameter inner fuel passage with a wall thickness of $1 \mathrm{~mm}$ surrounded by an

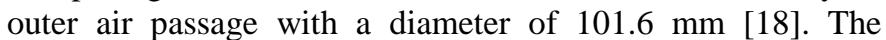
second flame that is modelled follows the work of Lee et al. [23], which uses the same burner as the Santoro flame, but methane instead of ethylene as the fuel. For the methane flame, the fuel velocity is $10.24 \mathrm{~cm} / \mathrm{s}$ while the air velocity is $11.94 \mathrm{~cm} / \mathrm{s}$ [24]. The computational domain consists of a nonuniform axisymmetric mesh of $384 \mathrm{CVs}$ in the axial direction and $150 \mathrm{CVs}$ in the radial direction for both of the modelled flames as the burner dimensions are the same.

\section{AliPHATIC COLLISION MECHANISM}

For the mechanism, it is assumed that aliphatic molecules collide together and under the right conditions may stick together. These conditions are accounted for in the collision efficiency that is prescribed in the CoFlame code. This mechanism was developed to function in a similar manner to particle coalescence, which has been implemented in the CoFlame code and validated. The primary difference between this mechanism and particle coalescence being the specific molecules that are colliding and contributing to soot inception. Particle coalescence uses large PAHs for inception, such as Benzo-a-pyrene, whereas this mechanism uses specific aliphatic species for the same means. A value is given to the collision efficiency to indicate that only $\mathrm{X}$ in every $\mathrm{Y}$ collisions will result in effective sticking. For example, if the collision efficiency is set 0.01 , this indicates that 1 in every 100 collisions will result in sticking, and the other 99 will result in rebound. Once the molecules stick together, they are treated as an incipient soot particle. This in effect means that this mechanism is contributing to the inception of soot, in addition to traditional PAH routes. Furthermore, this collision efficiency is highly dependent on molecular dynamics. Each of the aliphatic molecules considered has a radius and a concentration. The size and concentration inherently have an influence on the soot mass growth as the number of collisions increases with an increase in either one of those two parameters. It is important to note that the purpose of this mechanism is to simply link the aliphatics in the gas phase to the incipient solid soot phase, so as to assess their potential impact on soot mass growth and spatial distribution. The rate of inception is calculated according to kinetic theory:

$$
\partial \mathrm{N} / \partial \mathrm{t}=\beta \sqrt{ }\left[\left(8 \pi \mathrm{k}_{\mathrm{B}} \mathrm{T}\right) / \mu_{\mathrm{AB}}\right]\left(\mathrm{r}_{\mathrm{A}}+\mathrm{r}_{\mathrm{B}}\right)^{2} \mathrm{~A}_{\mathrm{V}}^{2}[\mathrm{~A}][\mathrm{B}]
$$

where $\beta$ is the collision efficiency, $\mathrm{k}_{\mathrm{B}}$ is the Boltzmann constant, $A_{V}$ is Avogadro's number, $\mu_{A B}$ is the collisional reduced mass for the two colliding aliphatics, $r_{A}$ and $r_{B}$ are the radii of the two colliding aliphatics, and [A] and [B] are the concentrations of the two colliding aliphatics [25].

The present study implements a 94 species mechanism, which models ethylene or methane combustion and PAH growth [16]. Some of the aliphatic species have been filtered out of use in the inception model based on their concentrations and carbon mass. Extremely low concentrations with low carbon mass such as $\mathrm{CH}_{2}$ have been removed from the proposed mechanism. The results of this analysis have determined that only 6 species of aliphatics are of particular interest in the present study. Those species are: $\mathrm{CH}_{4}$ (methane), $\mathrm{C}_{2} \mathrm{H}_{2}$ (acetylene), $\mathrm{C}_{2} \mathrm{H}_{6}$ (ethane), $\mathrm{C}_{3} \mathrm{H}_{6}$ (propene), $\mathrm{C}_{4} \mathrm{H}_{6}$ (butyne), and lastly $\mathrm{C}_{3} \mathrm{H}_{8}$ (propane).

While the present model does not represent the complete physics behind the problem, it can be used to determine the potential for mass transfer from the aliphatic gas phase to the soot solid phase. This strategy allows for analysis to be carried out, such that relationships can be made between the aliphatic species and soot mass growth. Adjusting the collision efficiency of aliphatics, and further adjustment to the soot surface reactivity parameter, as was done in [11] is a key component to this exercise. The present analysis has shown that the soot volume fraction, with respect to the proposed mechanism, can be tuned through the collision efficiency. Increasing the efficiency, leads to more soot growth whereas decreasing it has the opposite effect. Through this adjustment, it is hypothesized that the centerline soot volume fraction can be modified to better predict experimental values along the centerline of laminar diffusion flames.

The surface reactivity is a value that ranges from 0 to 1 and is representative of the portion of a soot particle's surface area that is available for chemical reaction. CoFlame allows for this surface reactivity to be modelled as either a constant or a function of temperature history. In the present study, it is taken as a constant value. This parameter is a factor in determining 
HACA growth and oxidation. Decreasing the surface reactivity normally leads to a decrease in the soot volume fraction as the HACA mechanism's contribution to surface growth decreases. Further adjustment of the surface reactivity, beyond what is already provided in literature $[11,26]$ comes in when a new growth mechanism is introduced into the numerical model as is the case in the present study.

\section{RESULTS AND DISCUSSION}

The generation of the model was predicated on being able to observe a positive influence that aliphatic-collision based inception would have on soot formation along the centerline of a laminar diffusion flame. It was not clear a priori if drawing aliphatics out of the gas phase for soot inception would reduce the amount of carbon available for PAHs or HACA growth thereby reducing soot concentrations in some locations of the flame. In order for the model to have some significance it had to be able to increase the soot volume fraction along the centerline while the wings remained close to the experimental peaks for the NSII flame. If that condition could not have been met then the model would not have a positive correlation between aliphatic collision based inception and soot distribution.

Figure 1 shows the predicted soot volume fraction along the pathline of maximum soot of the NSII flame as a function of height above the burner for varying collision efficiencies. The data are compared to a model without aliphatic inception and to experimental results. The solid horizontal line and gray band in Figure 1 at $9.7 \mathrm{ppm}$ indicates the experimentally measured peak value and associated uncertainty. It can be seen from Figure 1 that the predictive capability along the wings of the flame is generally unaffected by the addition of aliphatic inception. Furthermore, only collision efficiencies less than 1e-10 can lead to reasonable results. Adding aliphatic inception, however, shifts the location of peak soot formation to higher heights above the burner.

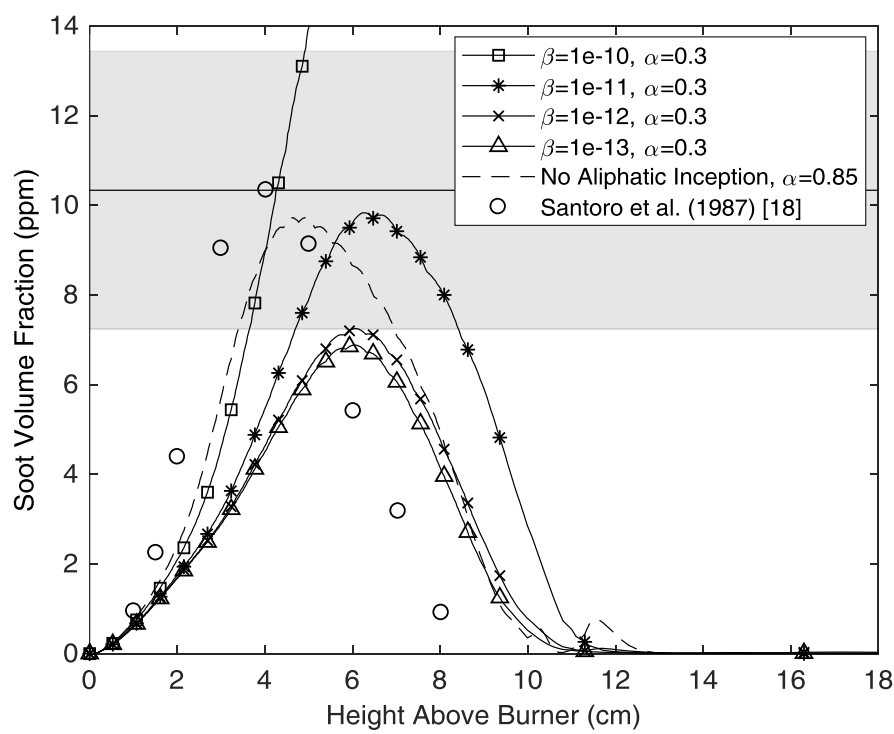

Figure 1. Predicted soot volume fraction as a function of height above the burner for varying collision efficiencies from 1e-10 to 1e-13 with constant surface reactivity of 0.3 along the wings of the NSII flame. The results of a model without aliphatic inception with $\alpha=0.85$ is shown alongside experimental results from [18]. The horizontal black line and gray band denote the experimentally measured peak.

Figure 2 shows the predicted soot volume fraction along the centerline of the NSII flame as a function of height above the burner for the same varying collision efficiencies. Here too the data are compared to a model without aliphatic inception and to experimental results. Again, only collision efficiencies less than 1e-10 lead to physically realistic results. Otherwise, implementing aliphatic based inception results in only a modest increase of soot volume fraction along the flame centerline. These figures illustrate that when $\alpha$ is set to 0.3 and $\beta$ is set to $1 \mathrm{e}-11$ there is an increase in the soot volume fraction along the centerline while the wings remain close to the experimental peaks. The same upward peak shifting phenomenon can be observed along the centerline in Figure 2. Also, the results of varying the collision efficiency and surface reactivity show that for the NSII flame, $\alpha$ can be lowered to a more physically realistic [27] value in order to correctly predict the wings peak soot volume fraction. In order to obtain results close to the experimental peaks for the NSII flame using CoFlame without an aliphatic collision based inception model, $\alpha$ had to be set to 0.85 .

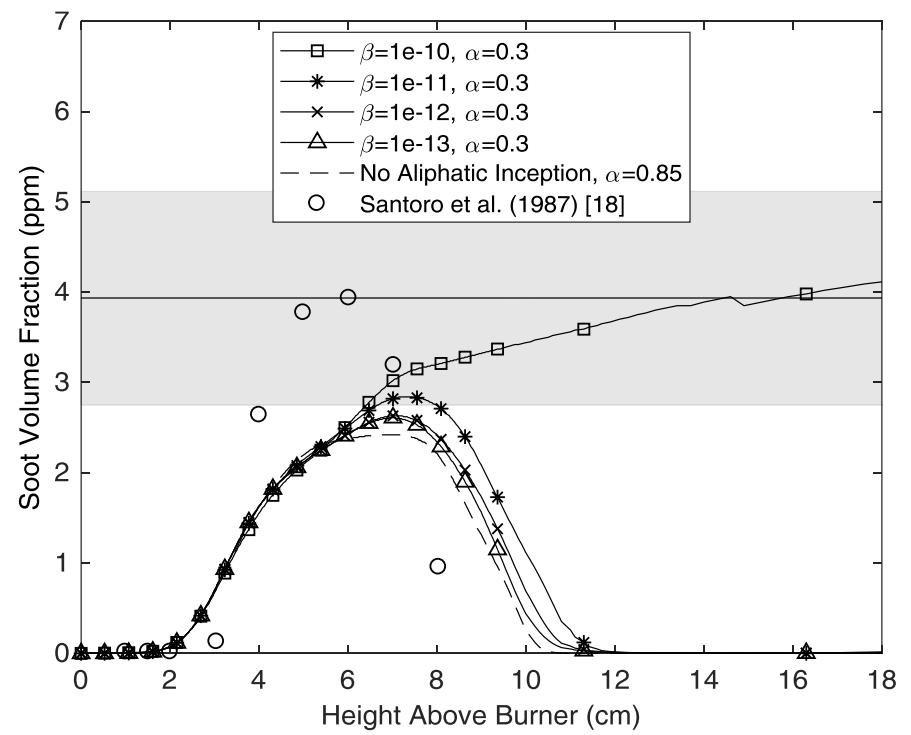

Figure 2. Predicted soot volume fraction as a function of height above the burner for varying collision efficiencies from 1e-10 to $1 \mathrm{e}-13$ with a constant surface reactivity of 0.3 along the centerline of the NSII flame. The results of a model without aliphatic inception with $\alpha=0.85$ is shown alongside experimental results from [18]. The horizontal black line and gray band denote the experimentally measured peak.

Similarly, the predicted soot volume fraction of the methane flame as a function of height above the burner for varying collision efficiencies and surface reactivity compared to a model without aliphatic inception and experimental results is shown in Figures 3 and 4. The results along the wings are shown in Figure 3 of the methane flame while Figure 4 shows the data along the centerline. The methane flame serves as a good benchmark to test the aliphatic collision based inception model for several reasons. The first and foremost reason is that 
methane flames are known to be dominated by inception over surface growth through the HACA mechanism as the concentration of acetylene throughout the flame is lower compared to ethylene combustion [12]. Since methane is a single carbon fuel, much less acetylene is formed when it is burned, leaving less opportunity to achieve accurate model predictions by refining HACA rates. Secondly, current soot models typically under predict the soot volume fraction along both the centerline and the wings in methane flames [12]. Using the same value for $\alpha$ and $\beta$ as for the NSII flame that achieved the aforementioned condition, 0.3 and $1 \mathrm{e}-11$ respectively, resulted in the under prediction of the soot volume fraction in both the centerline and wings. In ethylene flames, the surface reactivity can compensate for deficiencies in the chemical kinetic mechanism [12]. However, since methane flames are less influenced by HACA growth, the deficiencies in the reaction scheme remain prevalent. The latest version of CoFlame has added reactions specifically for methane flames as detailed by Chernov et al. [12]. Although there is an improvement in the model's predictive capability, increasing soot concentrations, even when the surface reactivity is set to its theoretical limit of unity, both regions of the flame remain under predicted. When the aliphatic collision based inception is applied with the same $\beta$ of $1 \mathrm{e}-11$ as before and $\alpha$ is increased to 1 , the theoretical maximum, there is an increase in the soot volume fraction along both the centerline and wings. Once again, the same peak shifting phenomenon can be observed. The aliphatic collision based inception model is able to improve the comparisons to experimental data, but not rectify all remaining inaccuracies in the methane flame.

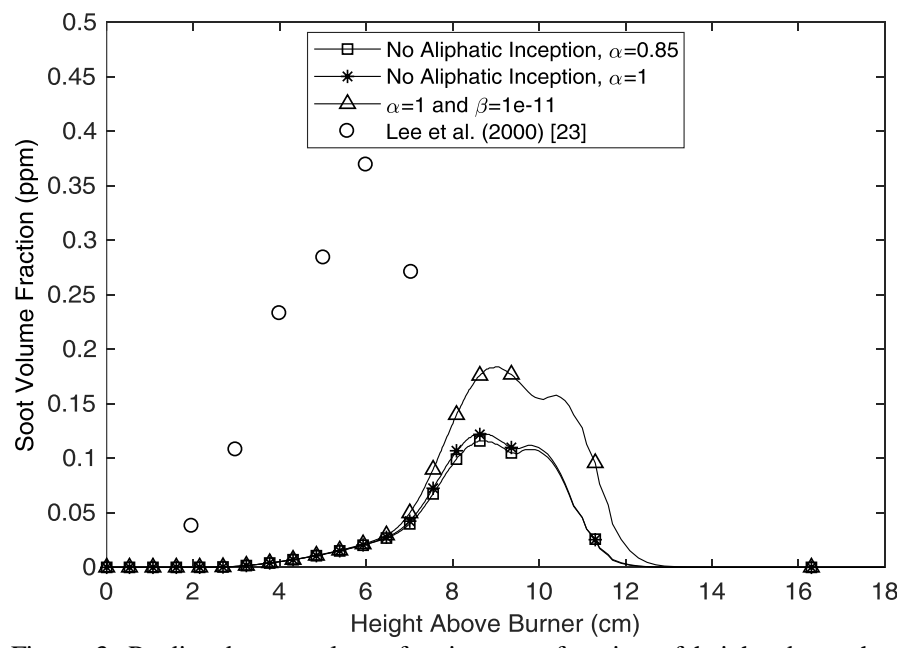

Figure 3. Predicted soot volume fraction as a function of height above the burner for a model without aliphatic inception using $\alpha$ of 0.85 and 1.0 along the wings of the methane flame. The aliphatic inception model is shown using the same $\beta$ as for the NSII, 1e-11. The experimental work of [20] is plotted.

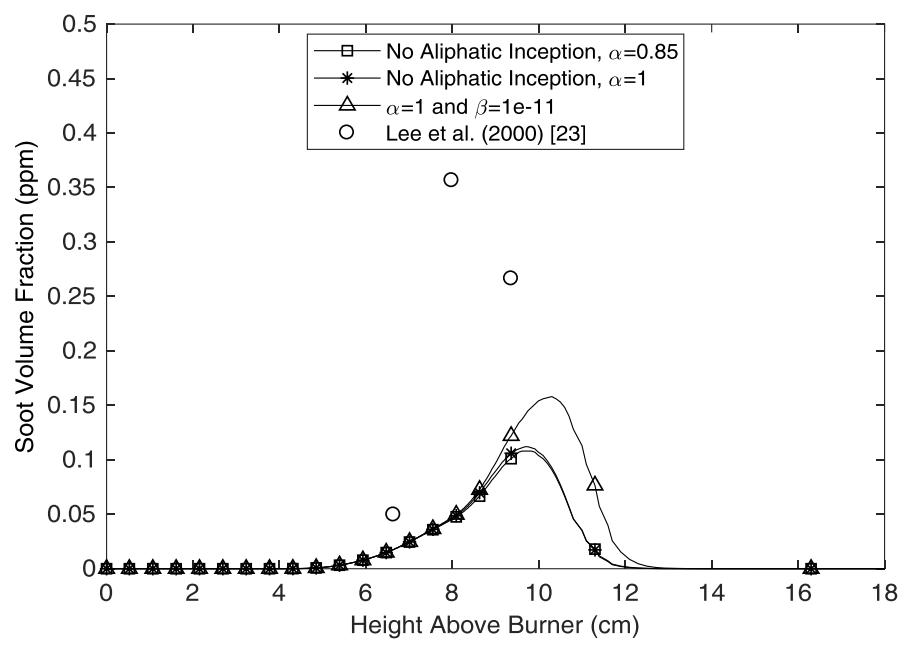

Figure 4. Predicted soot volume fraction as a function of height above the burner for a model without aliphatic inception using $\alpha$ of 0.85 and 1.0 along the centerline of the methane flame. The aliphatic inception model is shown using the same $\beta$ as for the NSII, 1e-11. The experimental work of [20] is plotted.

\section{SUMMARY AND CONCLUSIONS}

A new collision based inception mechanism was developed using an existing framework for larger PAHs but applied to aliphatics. The aliphatic inception mechanism was combined with PAH inception and implemented in the CoFlame code. The influence that an aliphatic-collision based inception model would have on the soot volume fraction distribution for coflow ethylene/air and methane/air laminar diffusion flames was investigated. It was found that for the ethylene flame, a surface reactivity, $\alpha$ of 0.3 and a collision efficiency, $\beta$ of $1 \mathrm{e}-11$ resulted in an increase in the peak soot volume fraction along the centerline, better predicting experimental values, while the predicted peak soot volume fraction along the wings was not degraded. For the methane flame, using the same $\beta$ of $1 \mathrm{e}-11$ resulted in an increase in both the centerline and wings of the flame as compared to using the same $\alpha$ in a soot model without aliphatic-collision based inception. Future work remains to test the applicability of the model to other combustion systems, in particular those for which model predictions do not completely explain or characterize experimental data.

To test the validity of the model further, the results will be expanded to other flame systems in order to ensure applicability to a wide variety of flames. The results of the mechanism need to be applied to the other pure ethylene/air diffusion flames studied by Santoro et al. [17, 18] to observe the effects this mechanism would have in those systems. The other ethylene flames suffer from the same predictive discrepancies as the NSII flame. A potential pathway of interest may also be to consider aliphatic molecule condensation and determine if it plays a significant role in soot formation. The current model acts as a proof of concept in that transfer of carbon mass from the aliphatic gas phase to soot particles seems to significantly improve the model's predictive capability in the centerline region of the flames studied, without degrading the HACA growth dominated wings. 


\section{ACKNOWLEDGEMENTS}

The authors thank Dr. Nadezhda Slavinskaya and Prof. Uwe Riedel of the German Aerospace Center (DLR) for providing the chemical reaction mechanism, thermodynamic data, and transport data for ethylene combustion and PAH formation. The authors acknowledge the Natural Sciences and Engineering Research Council of Canada (NSERC) for financial support. This research was enabled in part by support provided by Sharcnet of Compute Canada.

\section{REFERENCES}

[1] N. A. Janssen et al. Health Effects of Black Carbon, World Health Organization (2012).

[2] N. A. H. Janssen et al. Environmental Health Perspectives, 119 (12) (2012) 1691-1699.

[3] S. Vedal. Ambient Paraticles and Health: Lines that Divide, Journal of the Air and Waste Management Association, 47(5) (1997) 551-581.

[4] J. Hansen and L. Nazarenko. Soot Climate Forcing via Snow and Ice Albedos, Proceedings of the National Academy of Sciences of the United States of America, 101(2) (2004) 423-428.

[5] J. Penga et al. Proceedings of the National Academy of Sciences, 113 (16) (2016) 4266.

[6] B. S. Haynes, G. H. Wagner. Soot Formation, Progress in Energy and Combustion Science, 7(4) (1981) 229-273.

[7] M. Fairweather, W. P. Jones, H. S. Ledin, and R. P. Lindstedt. Prediction of Soot Formation in Turbulent, NonPremixed Propane Flames, Proceedings of the Combustion Institute, 24 (1992) 1067-1074.

[8] I. M. Kennedy. Models of Soot Formation and Oxidation, Progress in Energy and Combustion Science, 23(2) (1997) 95132.

[9] M. Frenklach and H. Wang. Detailed Modelling of Soot Particle Nucleation and Growth, Symposium (International) on Combustion, 23(1) (1991) 1559-1566.

[10] V. Chernov, Q. Zhang, M. J. Thomson, and S. B. Dworkin. Numerical Investigation of Soot Formation Mechanism in Partially-Premixed Ethylene-Air Co-Flow Flames, 159 (9) (2012) 2789.

[11] S. B. Dworkin, Q. Zhang, M. J. Thomson, and N. A. Slavinskaya, U. Riedel. Application of an Enhanced PAH Growth Model to Soot Formation in Laminar Coflow Ethylene/air Diffusion Flame, Combustion and Flame, 158(9) (2011) 1682-1695.

[12] V. Chernov, M. J. Thomson, S. B. Dworkin, and N. A. Slavinskaya, U. Riedel. Soot Formation with C1 and C2 Fuels using an Improved Chemical Mechanism for PAH Growth, Combustion and Flame, 161(2) (2014) 592.

[13] J. Appel, H. Bockhorn and M. Frenklach. Kinetic Modeling of Soot Formation with Detailed Chemistry and Physics: Laminar Premixed Flames of C2 Hydrocarbons, Combustion and Flame, 121(1) (200) 122-136.

[14] N. M. Marinov, W. J. Pitz, C. K. Westbrooke, A. M. Vincitore, M. J. Castaldi, and S. M. Senkan. Aromatic and Polycyclic Aromatic Hydrocarbon Formation in a Laminar
Premixed n-Butane Flame, Combustion and Flame, 114(1) (1998) 192-213.

[15] H. Wang. Formation of Nascent Soot and Other Condensed-Phase Materials in Flames, Proceedings of the Combustion Institute, 33(1) (2011) 41-67.

[16] N. A. Eaves, Q. Zhang, F. Liu, H. Guo, S. B. Dworkin, and M. J. Thomson. CoFlame: A Refined and Validated Numerical Algorithm for Modeling Sooting Laminar Coflow Diffusion Flames, Computer Physics Communications, 207 (2016) 464-477.

[17] R.J. Santoro, H.G. Semerjian, and R.A. Dobbins. Soot Particles Measurements in Diffusion Flames, Combustion and Flame 51 (1983) 203-218.

[18] R.J. Santoro, T.T. Yeh, J.J. Horvath, and H.G. Semerjian. The Transport and Growth of Soot Particles in Laminar Diffusion Flames, Combustion Science Technology, 53 (1987) 89-115.

[19] N.A. Eaves, A. Veshkini, C. Riese, Q. Zhang, S.B. Dworkin, and M.J. Thomson. A Numerical Study of High Pressure, Laminar, Sooting, Ethane-Air Coflow Diffusion Flames, Combustion and Flame 159 (2012) 3179-3190.

[20] F. Liu, H. Guo, G.J. Smallwood, and Ö.L. Gülder. Numerical Modelling of Soot Formation and Oxidation in Laminar Coflow Non-Smoking and Smoking Ethylene Diffusion Flames, Combustion Theory and Modelling, 7 (2003) 301-315.

[21] C.M. Megaridis and R.A. Dobbins. Soot Aerosol Dynamics in a Laminar Ethylene Diffusion Flame, Proceedings of the Combustion Institute, 22 (1988) 353-362.

[22] C.M. Megaridis and R.A. Dobbins. Comparison of Soot Growth and Oxidation in Smoking and Non-Smoking Ethylene Diffusion Flames, Combustion Science Technology, 66 (1989) 1-16.

[23] K. Lee, C. M. Megaridis, S. Zelepouga, A. V.Saveliev, L. A. Kennedy, O. Charon, and F. Ammourid. Soot Formation Effects of Oxygen Concentration in the Oxidizer Stream of Laminar Coannular Nonpremixed Methane/Air Flames, Combustion and Flame, 121(1) (2000) 323-333.

[24] A. Khosousi and S. B. Dworkin. Detailed Modelling of Soot oxidation by $\mathrm{O} 2$ and $\mathrm{OH}$ in Laminar Diffusion Flames, Proceedings of the Combustion Institute, 35(2) (2015) 19031910.

[25] M. Saffaripour, A. Veshkini, M. Kholghy, and M. J. Thomson. Experimental Investigation and Detailed Modeling of Soot Aggregate Formation and Size Distribution in Laminar Coflow Diffusion Flames of Jet A-1, a Synthetic Kerosene, and n-decane, Combustion and Flame, 161(3) (2014) 848-863. [26] A. Veshkini, S. B. Dworkin, and M. J. Thomson. Understanding Soot Particle Size Evolution in Laminar Ethylene/Air Diffusion Flames Using Novel Soot Coalescence Models, Combustion Theory and Modelling, 20(4) (2016) 707-734.

[27] A. Veshkini, S. B. Dworkin, M. J. Thomson. A Soot Particle Surface Reactivity Model Applied to a Wide Range of Laminar Ethylene/Air Flames, Combustion and Flame. 161(12) (2014) 3191-3200. 